\title{
On the polaron asymptotics at finite coupling constant
} LUIGI ACCARDI

Centro Vito Volterra

Università degli Studi di Roma

"Tor Vergata"

Stefano Olla

Dipartimento di Matematica

Università degli Studi di Roma

"Tor Vergata"

\section{Motivation and physical background}

A Polaron is an electron in a ionic crystal coupled with the lattice vibration field produced by itself through polarizaton of the crystal. The problem is to compute the energy of the ground state of the Polaron under the assumptions (due to Fröhlich, cf. [8]) that the atomic structure of the crystal can be neglected; that it is possible to tract it as a continuum dielectric; and that the only phonon waves that interact with the electron have the same frequency. Under these assumptions the Fröhlich hamiltonian for the polaron (with all physical constants equal to 1 ) is:

$$
H_{F}(\alpha)=\frac{1}{2} p^{2}+\sum_{k} a_{\bar{k}}^{+} a_{\bar{k}}+i(\sqrt{2} \alpha \pi)^{1 / 2} \sum_{k} \frac{1}{|\bar{k}|}\left(a_{\bar{k}}^{+} e^{-i \bar{k}^{\prime} \bar{x}}-a_{\bar{k}} e^{i \bar{k}^{\prime} \bar{x}}\right)
$$

where $\bar{x}$ is the vector position of the electron, $\bar{p}$ its conjugate momentum, $a_{\bar{k}}^{+}$ and $a_{\bar{k}}$ are the creation and annihilation operators of a phonon of momentum $\bar{k}$, and $\alpha$ is the coupling constant between the electron and the phonon field which depends on the dielectric constants of the ionic crystal (in $\mathrm{NaCl} \alpha$ is about 5 , and in general it runs from about 1 to 20 ). 
The lowest eigenvalue $E_{0}(\alpha)$ of $H_{F}(\alpha)$ has been studied for many years by a lot of techniques. In the weak coupling limit ( $\alpha$ small) perturbation treatment of (1) gives good results (cf. [7]).

For the strong coupling limit $(\alpha \rightarrow+\infty)$ a conjecture due to Pekar (cf. [18]) suggest that

$$
E_{0}(\alpha) \underset{\alpha \rightarrow+\infty}{\sim}-\gamma_{p} \alpha^{2}
$$

where

$$
\gamma_{p}=-\inf _{\substack{\phi \in L^{2}\left(\mathbb{R}^{3}\right) \\\|\phi\|_{2}=1}}\left\{\frac{1}{2} \int_{\mathbb{R}^{3}}|\nabla \phi|^{2} d x-\frac{1}{\sqrt{2}} \iint \frac{\phi^{2}(x) \phi^{2}(y)}{|x-y|} d x d y\right\}
$$

The minimizing $\phi$ in (3) is the solution of the non-linear Shrödinger equation

$$
-\frac{1}{2} \Delta \phi(x)-2 \sqrt{2}\left(\int \frac{|\phi(y)|^{2}}{|x-y|} d y\right) \phi(x)=e_{\phi}(x)
$$

which describes an electron trapped in its own hole. In [15] Lieb has shown that the minimizing $\phi$ in (3) exists and is unique up to translations (the problem was non trivial because of the non-convexity of the functional in (3)). In particular Lieb proved that the minimizing solution is infinitely differentiable and goes to zero at infinity. By numerical computations (cf. [17]) $\gamma_{p} \cong 0.108513$, very close to $\frac{1}{3 \pi}$ that is the value obtained in (3) when one uses gaussian functions as trial functions.

For intermediate coupling the most important techniques are those developed by Gross (cf. [10], who starts from the classical Riesz variational method) and of Feynman (cf. [6] and [7]), who reduced the problem to the asymptotic evaluation of a path integral. Using variational methods (cf. [7]) he obtains numerical results for an upper bound for $E_{0}(\alpha)$, which are still the best known bounds up to some improvements due to Luttinger and $\mathrm{Lu}$ (cf. [16]) who take into account the second order correction in Feynman's method.

Interesting direct numerical computations of the path integral were also performed using Monte Carlo techniques (cf. [9]).

The problem of the existence of a phase transition is first mentioned by Gross [10] who observers that the wave function of the polaron must be extended in the weak coupling region and localized for strong couplings. This corresponds to the intuitive idea that for $\alpha$ tending to zero the polaron 
dynamics tends to that of a free particle, and for $\alpha \rightarrow \infty$ a self localization effect arises.

The conjecture of Gross is that the transition between the localized and the extended function is abrupt, and this gives a discontinuity on the first derivative of $E_{0}(\alpha)$. The existence of such phase transition is an open problem at the moment.

In [16] Luttinger and Lu observe that in Feynman's harmonic approximation (an upper bound), a phase transition at $\alpha=5.8$ exists (Feynman knew about the discontinuity at 5.8, cf. [6]; but he considered this rather a disadvantage of the approximation that a result).

The same result is also obtained by Gross in [10]. But it is not clear if this dynamical instability is just a property of $H_{F}(\alpha)$ or if it comes from the approximation introduced to obtain the estimate. Another conjecture, given by Lepine and Metz (cf. [13], 1979), is that there might be two phase transition points. One from an extended translational invariant wave function to a two dimensional localized wave function. This conjecture is suggested by Fock approximation mean field theory applied to $H_{F}(\alpha)$.

All these results concern only upper bounds for $E_{0}(\alpha)$, while the physical literature about lower bounds is very poor (cf. [11] and [14]) and limited to a short range of $\alpha$.

\section{Mathematical formulation of the problem}

Using their powerful theory of large deviations for Markov processes [4], Donsker and Varadhan [5], found for $E_{0}(\alpha)$ the variational formula:

$$
-E_{0}(\alpha)=g(\alpha)=\sum_{Q \in M_{s}(\Omega)}\left\{2 \alpha Q\left(\int_{0}^{\infty} \frac{e^{-\sigma} d \sigma}{|w(\sigma)-w(0)|}\right)-H\left(Q \mid E_{0]}^{w}\right\}\right.
$$

where $\Omega=D\left(\mathbb{R}_{+}, \mathbb{R}^{3}\right)$ is the space of all right-continuous functions $\mathbb{R}_{+} \rightarrow \mathbb{R}^{3}$ with only first kind discontinuities and left limits; $M_{s}(\Omega)$ denotes the space of all the stationary measures on $\Omega ; w(t)$ denotes the map

$$
w(t): \omega \in \Omega \rightarrow w(t)(\omega)=\omega(t) \in \mathbb{R}^{3}
$$

$E_{0]}^{w}$ is the conditional expectation of the Wiener process onto the $\sigma$-algebra at time zero; and $H\left(Q \mid E^{w}\right)$ is the entropy of $Q$ with respect to $E_{0]}^{w}$-defined in [4] (cf. also [1]). 
Using formula (1), Donsker and Varadhan were able to give a rigorous proof of Pekar's conjecture 5, i.e. the validity of (2) and (3).

In the present paper we want to exploit the identity (1) to study the behaviour of $E_{0}(\alpha)$ at finite $\alpha$ and the problem of the existence of a phase transition. At the moment we have not a full proof of the existence of a phase transition (cf. the remark at the end of section (3.)), however we obtain the following results:

i) We obtain a rigorous derivation of the results obtained in the physical literature. In particular we show that the expression obtained by Feynman [6], [1], Gross [10], Luttinger - Lu [16], in the harmonic approximation for $E_{0}(\alpha)$ is obtained from (1) by restriction of the sup to the Ornstein-Uhlenbeck proceses (cf. section (5.)).

ii) We improve these estimates, deriving an explicit finite dimensional variational formula for the restriction of the sup in (1) to all distorted brownian motions (cf. section (3.)) and to all stationary gaussian processes (cf. section (4.)).

iii) We obtain a general lower estimate for $E_{0}(\alpha)$ (upper estimate for $g(\alpha)$ ) which seems not to be present in the physical literature, and which allows to obtain both theoretical and numerical informations on the validity of the upper estimate (cf. the end of section (3.)).

Notational remark: following Donsker and Varadhan's notations we deal with $g(\alpha)$ rather than $E_{0}(\alpha)(=-g(\alpha))$. So to compare our results with the ones mentioned in section (1.) one should keep in mind that the upper estimates in these ones correspond to our lower estimate, and conversely. Moreover, in the identity (1) we have rescaled $\alpha$ by a factor $\sqrt{8}$. Thus, to derive the numerical results in the physical literature from our ones, one should multiply $\alpha$ by the factor $\sqrt{8}$.

\section{The Markovian approximation}

Let us first recall the statement of Donskier-Varadhan's "contraction principle" (cf. [4]) namely: for any probability measure $m$ on $\mathbb{R}^{3}$, one has:

$$
\inf _{\substack{Q \in M_{s}(\Omega) \\ Q_{0}=m}} H\left(Q \mid E^{w}\right)=I_{w}(m)
$$


where $Q_{0}$ is the marginal distribution of $Q \in M_{s}(\Omega)$, and:

$$
I_{w}(m)= \begin{cases}\int_{\mathbb{R}^{3}} \frac{[\nabla f]^{2}}{f}(x) d x & \text { if } m(d x) \ll d x \\ +\infty & \text { and } f=\frac{d m}{d x} \in C^{\prime}\left(\mathbb{R}^{3}\right) \\ +\infty & \text { otherwise }\end{cases}
$$

Denote

$$
H=\left\{\phi \in L^{2} \cap \mathcal{C}^{2}\left(\mathbb{R}^{3}\right),\|\phi\|_{L^{2}}=1,\|\nabla \phi\|_{L^{2}}<+\infty\right\}
$$

where $\nabla$ denotes the gradient.

In Appendix (A1) it is shown that, denoting for each $\phi \in H, Q^{\phi} \in M_{s}(\Omega)$ the stationary Markovian measure with transition semi-group generated by

$$
L_{\phi}=+\frac{\Delta}{2}+\frac{\nabla \phi}{\phi} \cdot \nabla
$$

and with initial distribution $\phi^{2}(x) d x$ (the associated Markov process is called in the literature a "distorted Brownian Motion" (cf. for example [3]), then:

$$
H\left(Q^{\phi} \mid E_{0]}^{w}\right)=\int_{\mathbb{R}^{3}}|\nabla \phi|^{2} d x
$$

namely the inf in (1) is realized by $Q^{\phi}$ for $m(d x)=\phi^{2}(x) d x$.

Therefore, restricting the sup in (1) to the class $\left\{Q^{\phi}, \phi \in H\right\} \subseteq M_{s}(\Omega)$ we obtain the following lower estimate:

Theorem $1 \forall \alpha>0$

$$
g(\alpha) \geq \sup _{\phi \in H}\left\{\alpha \int_{\mathbb{R}^{3} \times \mathbb{R}^{3}} \int\left(1-\frac{\Delta}{2}+\frac{\Delta \phi}{2 \phi}(x)\right)^{-1}(x, y) \frac{\phi(x) \phi(y)}{|x-y|} d x d y-\int_{\mathbb{R}^{3}}\left|\nabla \phi^{2}\right| d x\right\}
$$

Remark 1 It is possible to give a simple direct proof of the lower estimate (6) which does not make use of the full technical apparatus developed by Donsker and Varadhan. We will not include this here for lack of space.

Proof. From (1) and (6) it follows that:

$$
\begin{aligned}
g(\alpha) & =\sup _{Q \in M_{s}(\Omega)}\left\{2 \alpha Q\left(\int_{0}^{\infty} \frac{e^{-\sigma} d \sigma}{|w(\sigma)-w(0)|}\right)-H\left(Q \mid E^{w}\right)\right\} \geq \\
& \geq \sup _{\phi \in H}\left\{2 \alpha Q^{\phi}\left(\int_{0}^{\infty} \frac{e^{-\sigma} d \sigma}{|w(\sigma)-w(0)|}\right)-\int_{\mathbb{R}^{3}}|\nabla \phi|^{2} d x\right\}
\end{aligned}
$$


The density of the marginal distribution of $Q^{\phi}$ is just $\phi^{2}(x)$, then by Fubini Tonelli's theorem:

$$
\begin{aligned}
& Q^{\phi}\left(\int_{0}^{\infty} \frac{e^{-\sigma} d \sigma}{|w(\sigma)-w(0)|}\right)= \\
= & \int_{\mathbb{R}^{3}} \phi^{2}(x) d x \int_{0}^{\infty} e^{-\sigma} e^{+\sigma L} \phi\left(\frac{1}{|\cdot-x|}\right) d \sigma= \\
= & \int_{\mathbb{R}^{3} \times \mathbb{R}^{3}} \int\left[1-M_{\phi} L_{\phi} M_{\phi}^{-1}\right]^{-1}(x, y) \frac{\phi(x) \phi(y)}{|x-y|} d x d y
\end{aligned}
$$

were $\left(1-L_{\phi}\right)^{-1}(x, y)$ is the kernel of the resolvent $\left(1-L_{\phi}\right)^{-1}$ in $L^{2}\left(\mathbb{R}^{3}, \phi^{2}(x) d x\right)$.

Then by (8)

$$
\begin{aligned}
& Q^{\phi}\left(\int_{0}^{\infty} \frac{e^{-\sigma} d \sigma}{|w(\sigma)-w(0)|}\right)= \\
= & \iint_{\mathbb{R}^{3} \times \mathbb{R}^{3}} \phi(x)\left(1-L_{\phi}\right)^{-1}(x, y) \phi(y) \frac{\phi(x) \phi(y)}{|x-y|} d x d y= \\
= & \iint_{\mathbb{R}^{3} \times \mathbb{R}^{3}}\left[M_{\phi}\left(1-L_{\phi}\right)^{-1} M_{\pi}^{-1}\right](x, y) \frac{\phi(x) \phi(y)}{|x-y|} d x d y= \\
= & \iint_{\mathbb{R}^{3} \times \mathbb{R}^{3}}\left[1-M_{\phi} L_{\phi} M_{\phi}^{-1}\right]^{-1}(x, y) \frac{\phi(x) \phi(y)}{|x-y|} d x d y
\end{aligned}
$$

where $M_{\phi}: f \in L^{2}\left(\mathbb{R}^{3}, \phi^{2}(x) d x\right) \rightarrow \phi f \in L^{2}\left(\mathbb{R}^{3}, d x\right)$ denotes the operator of multiplication $\phi$, and $\left[1-M_{\phi} L_{\phi} M^{-1}\right]^{-1}(x, y)$ is the kernel of $\left[1-M_{\phi} L_{\phi} M_{\phi}^{-1}\right]^{-1}$ in $L^{2}\left(\mathbb{R}^{3}, d x\right)$. Since

$$
M_{\phi} L_{\phi} M_{\phi}^{-1}=\phi\left[+\frac{1}{2} \nabla+\frac{\nabla \phi}{\phi} \cdot \nabla\right] \phi^{-1}=+\frac{1}{2} \Delta-\frac{\Delta \phi}{2 \phi}
$$

(6) follows from (7), (9), (10).

In order to have an idea of how good the lower estimate (6) is, let us compare it with the upper estimate obtained as follows: start from Donsker and Varadhan's variational expression for $g(\alpha)$, i.e.:

$$
\begin{gathered}
g(\alpha)=\sup _{Q \in M_{s}(\Omega)}\left\{2 \alpha Q\left(\int_{0}^{\infty} \frac{e^{-\sigma} d \sigma}{|w(\sigma)-w(0)|}\right)-H\left(Q \mid E^{w}\right)\right\} \\
=\sup _{Q \in M \operatorname{erg}(\Omega), H\left(Q \mid E^{w}\right)}<\infty\left\{2 \alpha Q\left(\int_{0}^{\infty} \frac{e^{-\sigma} d \sigma}{|w(\sigma)-w(0)|}\right)-H\left(Q \mid E^{w}\right)\right\}
\end{gathered}
$$




$$
=\sup _{\phi \in H} \sup _{p(\cdot \cdot \cdot)}\left\{2 \alpha \int_{0}^{\infty} e^{-\sigma} d \sigma \int_{\mathbb{R}^{3}} \phi^{2}(x) d x \int_{\mathbb{R}^{3}} \frac{p(d y, \sigma \mid x, 0)}{|y-x|}-\infty_{[\phi, p]} H\left(Q \mid E^{w}\right)\right\}
$$

where we have used the following notations: for a stationay measure $Q$ with marginal $\phi^{2}(x) d x$ and locally absolutely continuous with respect to the Wiener measure, we denote $p(d y, \sigma \mid x, 0)$ the conditional probability of $Q \mid F_{0} \vee F_{\sigma}$ with respect to $F_{0}$; thus:

$$
Q\left(\frac{1}{|w(\sigma)-w(0)|}\right)=\int_{\mathbb{R}^{3}} \phi^{2}(x) d x \int_{\mathbb{R}^{3}} \frac{p(d y, \sigma \mid x, 0)}{|y-x|}
$$

Moreover, $\sup _{p(\cdot \cdot \cdot)}^{\prime}$ denotes the sup taken over all the transition probabilities obtained as above; and $\inf _{[\phi, p]}$ denotes the inf taken over all the stationary (ergodic) measures $Q$ whose marginal is $\phi^{2}(x) d x$ and whose associated transition probability density is $p$. With these notations we have the upper estimate:

$$
\begin{aligned}
g(\alpha) & \leq \sup \left\{2 \alpha \sup _{p} \int_{0}^{\infty} e^{-\sigma} d \sigma_{\mathbb{R}^{3}} \phi^{2}(x) d x \int_{\mathbb{R}^{3}} \frac{p(d y, \sigma \mid x, 0)}{|y-x|}-\inf _{Q_{0}=\phi^{2}(x) d x} H\left(Q \mid E^{w}\right)\right\} \\
& =\sup _{\phi \in H}\left\{2 \alpha\left(\sup _{p}^{\prime} \int_{0}^{\infty} e^{-\sigma} d \int_{\mathbb{R}^{3}} \phi^{2}(x) d x \int_{\mathbb{R}^{3}} \frac{p(d y, \sigma \mid x, 0)}{|y-x|}\right)-\int_{\mathbb{R}^{3}}|\nabla \phi|^{2} d x\right\}
\end{aligned}
$$

Thus the exactness of the lower estimate (6) would be implied by the fact that the sup of the integral

$$
\int_{0}^{\infty} e^{-\sigma} d \sigma \int_{\mathbb{R}^{3}} \phi^{2}(x) d x \int_{\mathbb{R}^{3}} \frac{p(d y, \sigma \mid x, 0)}{|y-x|}
$$

over all stationary ergodic transition probabilities $p(d y, \sigma \mid x, 0)$ is achieved on those of the type $\exp \sigma L_{\phi}(x, y) d y$, i.e. on the kernels of the semi-group of the distorted brownian motion associated to the functions $\phi \in H$. We conjecture that this "conditional contraction principle" is true, but at the moment we have not a complete mathematical proof of it.

\section{The Gaussian approximation}

More explicit computations are possible if we choose as class of trial processes the stationary gaussian processes. Also here the problem is reduced to a finite dimensional one, because we can take the supremum over all covariance functions. 
Let be $\rho_{i j}(t)$ the covariance matrix function of the stationary gaussian measure $Q^{\rho}$, corresponding to a stationary gaussian process $w(t)=\left(w_{1}(t)\right.$, $\left.w_{2}(t), w_{3}(t)\right): \omega \rightarrow \mathbb{R}$ characterized by the condition

$$
\left.Q^{\rho}\left(w_{i}(t+s) w_{j}^{\prime} s\right)\right)=\rho_{i j}(t) ; \quad s \in \mathbb{R}_{+} ; \quad j, i=1,2,3
$$

The process $y_{i}(t)=w_{i}(t)-w_{i}(0)(i=1,2,3)$ is then gaussian with covariance matrix

$$
\sigma_{i j}(t)=Q^{\rho}\left(y_{i}(t) y_{j}(t)\right)=2\left(\rho_{i j}(0)-\rho_{i j}(t)\right)
$$

Denoting $\left\{\lambda_{j}(t), j=1,2,3\right\}$ the eigenvalues of the matrix $\left(\sigma_{i j}(t)\right)$, after some elementary computations one finds:

$$
Q^{\rho}\left(\frac{1}{|w(t)-w(0)|}\right)=\frac{1}{(2 \pi)^{3 / 2}} \frac{1}{\left(\prod_{j=1}^{3} \lambda_{j}(t)\right)^{1 / 2}} \int_{\mathbb{R}^{3}} \frac{e^{-\frac{1}{2} \sum_{i=1}^{3} \lambda_{j}^{-1}(t) x_{j}^{2}}}{|x|} d x
$$

In particular, if $\lambda_{j}(t)=\lambda(t), j=1,2,3$, then:

$$
Q^{\rho}\left(\frac{1}{|w(t)-w(0)|}\right)=\frac{1}{(2 \pi \lambda(t))^{3 / 2}} \int_{\mathbb{R}^{3}} \frac{e^{-\frac{1}{2} \lambda^{-1}(t)|x|^{2}}}{|x|}=\left(\frac{2}{\pi \lambda(t)}\right)^{1 / 2}
$$

Using the well known formulae (cf. e.g. [12]) for the Radon-Nikodym derivatives of gaussian measure one can also give an explicit expression for the relative entropy of $Q^{\rho}$ with respect to the Wiener measure. For lack of space we do not discuss here the general case (cf. [12]) and limit ourselves to an important particular case, to be discussed in the next section.

\section{The harmonic approximation}

In the intersection of the two classes of processes considered respectively in section (3) and (4) one finds the Ornstein-Uhlenbeck processes, i.e. the stationary gaussian markovian process with covariance matrix funciton

$$
\rho_{i j}(t)=\frac{1}{2 \beta} e^{-\beta t} \delta_{i j} ; \quad i, j=1,2,3
$$

where $\beta>0$ is a parameter characteristic of the process.

The density of the invariant measure is

$$
\psi^{2}(x)=\left(\frac{\pi}{\beta}\right)^{-3 / 2} e^{-\beta x^{2}}
$$


So $\beta^{-1}$ is also the variance of the marginal distribution of the process.

The infinitesimal generator of the corresponding markovian semigroup is

$$
L_{\beta}=+\frac{1}{2} \Delta-\beta \cdot \nabla
$$

so it has the form (4) and therefore the Ornstein-Uhlenbeck processes belong to the class of markovian processes considered in $\S 3,(5)$ and (2)

$$
H\left(Q_{\beta}^{o u} \mid E^{w}\right)=\int_{\mathbb{R}^{3}}|\nabla \psi(x)|^{2} d x=\frac{3}{4} \beta
$$

In this case the matrix $\sigma_{i j}(t)$ defined by (2) is diagonal and we can put

$$
\begin{gathered}
\lambda(t)=\frac{1}{\beta}\left(1-e^{-\beta t}\right) \\
Q_{\beta}^{o u}\left(\frac{1}{|w(t)-w(0)|}\right)=\sqrt{\frac{2}{\pi}} \frac{1}{\left(1-e^{-\beta t}\right)^{1 / 2}}
\end{gathered}
$$

Then it is possible to compute explicitly also the action term in (1)

$$
Q_{\beta}^{\text {ou }}\left(\int_{0}^{\infty} \frac{e^{-\sigma} d \sigma}{|w(t)-w(0)|}\right)=\sqrt{\frac{2}{\pi}} \int_{0}^{\infty} \frac{e^{-t} d t}{\left(1-e^{-\beta t}\right)}=\delta 2 \beta^{1 / 2} \frac{\Gamma\left(1+\frac{1}{\beta}\right)}{\Gamma\left(\frac{1}{2}+\frac{1}{\beta}\right)}
$$

where $\Gamma$ is the gamma function.

Then using O.U. processes as trial processes in (1) one obtains the following lower estimate.

$$
g(\alpha) \geq \sup _{\beta}\left\{\alpha \beta^{1 / 2} \frac{\Gamma\left(1+\frac{1}{\beta}\right)}{\Gamma\left(\frac{1}{2}+\frac{1}{\beta}\right)}-\frac{3}{4} \beta\right\}
$$

The lower bound (8) is just that obtained by Feynman in [6], Gross in [10], Luttingen-Lu [16], with completely different approaches.

\section{Appendix (A1) Relative entropy of distort- ed Brownian motion}

Let $\phi \in \mathcal{C}^{2}\left(\mathbb{R}^{3}\right) \cap L^{2}(d x)$ such that $\|\phi\|_{L^{2}}=1$ and $\|\nabla \phi\|_{L^{2}}<+\infty$. Let $\left.Q^{\phi} \in M_{s} \Omega\right)$ the Markov process generated by

$$
L_{\phi}=\frac{1}{2} \Delta+\frac{\nabla \phi}{\phi} \cdot \nabla
$$


Let $E_{0}^{Q^{\phi}}$ the conditional expectation of $Q^{\phi}$ on the $\sigma$-algebra at time zero. Then by Cameron-Martin's formula and by Ito's formula:

$$
\frac{d E_{0}^{Q \phi} \mid F[0, t]}{d E_{0}^{w} \mid F[0, t]}=\frac{\phi\left(w_{t}\right)}{\phi\left(w_{0}\right)} \exp \left(-\frac{1}{2} \int_{0}^{t} \frac{\Delta \phi}{\phi}\left(w_{s}\right) d s\right)
$$

Then by [4] and by the stationarity of $Q^{\phi}$ :

$$
\begin{gathered}
H\left(Q^{\phi} \mid E_{0}^{w}\right)=Q^{\phi}\left(\lg \frac{d E_{0}^{Q^{\phi}} \mid F[0,1]}{d E_{0}^{w} \mid F[0,1]}\right)= \\
=Q^{\pi}\left(\lg \phi\left(w_{t}\right)-\lg \phi\left(w_{0}\right)-\frac{1}{2} \int_{0}^{t} \frac{\Delta \phi}{\phi}\left(w_{s}\right) d s\right)= \\
=-\frac{1}{2} \int_{0}^{1} Q^{\phi}\left(\frac{\Delta \phi}{\phi}\left(w_{s}\right)\right) d s= \\
=-\frac{1}{2} Q^{\phi}\left(\frac{\Delta \phi}{\phi}\left(w_{0}\right)\right)=-\frac{1}{2} \int_{\mathbb{R}^{3}} \frac{\Delta \phi}{\phi}(x) \phi^{2}(x) d x= \\
=-\frac{1}{2} \int_{\mathbb{R}^{3}} \phi \Delta \phi d x=\frac{1}{2} \int_{\mathbb{R}^{3}}|\nabla \phi|^{2} d x
\end{gathered}
$$

So we have the explicit formula:

$$
H\left(Q^{\phi} \mid E^{w}\right)=\frac{1}{2} \int_{\mathbb{R}^{3}}|\nabla \phi|^{2} d x
$$

\section{References}

1. L. Accardi, S. Olla.: Donsker and Varadhan theory for stationary processes, Preprint (1984).

2. J. Adamowski, B. Goerlach, H. Leschke: "Strong coupling limit of polaron energy", revisted Physics Letters 79A, number 2, 3, Sept. 1980 (249-251).

3. S. Albeverio, R. Hoegh-Krohn, L. Streit: "Energy forms, Hamiltonians, and distorted Brownian path", J. Math. Phys. 18 5, (1977) 907-917. 
4. M.D. Donsker, S.R.S. Varadhan: "Asymptotic evaluation of certain Markov process expectations for large time, IV", Comm. Pure Appl. Math. 36 (1983) 182-212.

5. M.D. Donsker, S.R.S. Varadhan: "Asymptotics for the Polaron", Comm. Pure Appl. Math. 36 (1983) 505-528.

6. R.P. Feynman: "Slow electrons in a polar crystal", Phys. Rev. 973 (1955) 660-665.

FeHi65 Feynman R.P., A.R.Hibbs: Quantum Mechanics and Path Integrals Mc Graw-Hill, INc. (1965)

7. R.P. Feynman: Statistical Mechanics, W.A. Benjamin, Reading, MA, 1972.

8. H. Fröhlich: "Electrons in lattice fields", Advan. Phys. 3 (1954) 325361.

9. I.M. Gelfand, N.M. Chentsov: "The numerical calculation of path integrals", J.E.T.P. 31 (1956) 1106-11107.

10. E.P. Gross: "Analytical methods in the theory of electron lattice interactions", Ann. Phys. 8 (1959) 78-99.

11. D.M. Larsen: "Upper and lower bounds for the intermdiate - Coupling Polaron Grand-State Energy", Phys. Rev. 172 (1968) 967-971.

12. I. Guikhman, A. Skorokhod: The theory of stochastic processes.

13. Y. Lepine, D. Metz: "Mean field theory of a single Fröhlic polaron (possible existence of phase transitions)", Phys. Sol. (b) 96 (1979) 797-806.

14. E.H. Lieb, K. Yamazaki: "Ground state energy and effective mass of the polaron", Phys. Rev. 1113 (1968) 728-733.

15. H. Lieb: "Existence and uniqueness of the minimizing solution of Choquard's nonlinear equation", "Studies Appl. Math." 57 (1977) 93-105.

16. J.M. Luttinger, Chih-Yuan Lu: "Generalized path-integral formalism of the polaron problem", Phys. Rev. B 2110 (1980) 4251-4263. 
17. S.J. Miyake: "Strong-coupling limit of the polaron ground state", J. Phys. Soc. Japan 381 (1975) 181-182.

18. S.I. Pekar: "Theory of polarons", Zh. Experim. i Tear. Fiz. 19 (1949) 796.

\section{Gaussian approximation}

Dalla (3)

$$
\begin{gathered}
\phi^{\prime}\left(\frac{1}{|w(t)-w(0)|}\right)=\frac{1}{(2 \pi)^{3 / 2}}\left(\frac{1}{\prod_{j=1}^{3} \lambda_{j}(t)}\right)^{1 / 2} \int_{\mathbb{R}^{3}} \frac{e^{-\frac{1}{2} \sum_{i=1}^{3} \lambda_{j}^{-1} x_{j}^{2}}}{|x|} d^{3} x= \\
\frac{2}{(2 \pi)^{3 / 2}}\left(\frac{1}{\prod_{j=1}^{3} \lambda_{j}(t)}\right)^{1 / 2} \int_{0}^{2 \pi} d \varphi \int_{0}^{1} \frac{d y}{f(\varphi)+\left(\lambda_{3}^{-1}-f(\varphi)\right) y^{2}}
\end{gathered}
$$

$\operatorname{con} f(\varphi)=\lambda_{1}^{-1} \cos ^{2} \varphi+\lambda_{2}^{-1} \sin ^{2} \varphi$

Caso a) $\lambda_{1}(t)=\lambda_{2}(t) \neq \lambda_{3}(t)$ (simmetria cilindrica)

$$
\begin{gathered}
f(\varphi)=\lambda_{1}^{-1}(t) \\
Q^{\rho}\left(\frac{1}{|w(t)-w(0)|}\right)=\frac{4 \pi}{(2 \pi)^{3 / 2}} \frac{1}{\lambda_{1}(t) \lambda_{3}^{1 / 2}(t)} \int_{0}^{1} \frac{d y}{\lambda_{1}^{-1}(t)-\left(\lambda_{3}^{-1}-\lambda_{1}^{-1}\right) y^{2}}= \\
=\sqrt{\frac{2}{\pi}} \frac{1}{\lambda_{3}^{1 / 2}(t)} \int_{0}^{1} \frac{d y}{1-\frac{\lambda_{3}^{-1}-\lambda_{1}^{-1}}{\lambda_{1}^{-1}} y^{2}}= \\
=\sqrt{\frac{3}{\pi}} \frac{1}{\left(\lambda_{1}(t)-\lambda_{3}(t)\right)^{1 / 2}} \operatorname{arctg}\left(\frac{\lambda_{1}(t)}{\lambda_{3}(t)}-1\right) \text { se } \lambda_{1}(t)>\lambda_{3}(t) \\
=\frac{1}{\sqrt{\pi}} \frac{1}{\left(\lambda_{3}(t)-\lambda_{1}(t)\right)^{1 / 2}} \lg \left(\frac{2 \lambda_{3}(t)}{\lambda_{1} 1+1}-1\right) \text { se } \lambda_{3}(t)>\lambda_{1}(t)
\end{gathered}
$$

Non sembra affetto semplice calcolare analiticamente

$$
\int_{0}^{\infty} e^{-t} Q^{\rho}\left(\frac{1}{|w(t)-w(0)|}\right) d t
$$


neanche nel caso di simmetria cilindrica e con

$$
\lambda_{j}(t)=\frac{1}{\beta_{1}}\left(1-e^{-\mu_{i} t}\right)
$$

che corrisponde al processo O.U.

$$
L=\frac{1}{2} \Delta-\beta x \cdot \nabla \quad \beta=\left(\begin{array}{ccc}
\beta & 0 & 0 \\
0 & \beta_{2} & 0 \\
0 & 0 & \beta_{3}
\end{array}\right)
$$

\section{Entropia dei processi gaussiani}

L.A. Shepp: Random Nykodym derivative of Gaussian measures - Ann. Math. Statist. 37 (1966)

Il risultato è essenzialmente questo: sia $\mu$ misura gaussiana con covarianza $R(s, t)$ e media 0 allora $\mu \sim \mu_{w}$ su $[0, T]$ ( $\mu_{w}$ misure di Wiener) se e solo se $\exists K \in L^{2}([0, T] \times[0, T])$ tale che

$$
\begin{gathered}
h(s, t)=s \wedge t-\int_{0}^{t} \int_{0}^{s} k(u, v) d u d v \\
\Leftrightarrow k(s, t)=-\frac{\partial}{\partial s} \frac{\partial}{\partial t} R(s, t)
\end{gathered}
$$

$\left(\right.$ se $h_{1}=\frac{\partial}{\partial s} h(s, t)$ è continua in $t$ per $\left.t \neq s \Rightarrow R_{1}(s, s+)-R_{1}(s, s-)=1\left({ }^{*}\right)\right)$ è facile vedere che o.u. soddisfa $(*)$.

La $\frac{d \mu}{d \mu_{u}}$ viene data in temini dalla risolvente dell'operatore $k$.

\section{$9 \quad$ Appendice non pubblicata}

Appendix (A2.). A direct proof of the estimate (6).

From Theorem (3.3) of Donsker and Varadhan [4] we know that

$$
g(\alpha)=\lim _{t \rightarrow \infty} \frac{1}{t} \log E_{0]}^{w}\left(\exp \alpha \int_{0}^{t} \int_{0}^{t} \frac{e^{-|\sigma-\tau|}}{|w(\sigma)-w(\tau)|} d \sigma d \tau\right)
$$

Now we know that, for any $\delta>0$ and uniformly in $t>0$, one has:

$$
\left|t P_{t} T_{[0, t]}\left(2 \alpha \int_{0}^{\infty} \frac{e^{-\sigma} d \sigma}{|w(\sigma)-w(0)|+\delta}\right)-\alpha \int_{0}^{t} \int_{0}^{t} \frac{e^{-|\sigma-\tau|}}{|w(\sigma)+w(\tau)|+\delta} d \sigma d \tau\right| \leq \frac{2 \alpha}{\delta}
$$


where $P_{t}$ is the periodization operator and $T_{[0, t]}$ is the occupation time operator defined respectively by (x.) and (x.) in [1].

From this it is easy to see that, for any $\delta>0$ :

$$
g(\alpha) \geq \liminf _{t \rightarrow \infty} \frac{1}{t} \log E_{0]}^{w}\left(\exp t 2 \alpha P_{t} T_{[0, t]}\left(\int_{0}^{\infty} \frac{e^{-\sigma} d \sigma}{|w(\sigma)-w(0)|+\delta}\right)\right)
$$

Therefore, in the notations of section (3) and of Appendix (A1), and for $\varphi \in \mathcal{H}$ one has:

$$
g(\alpha) \geq \liminf _{t \rightarrow \infty} \frac{1}{t} \log E_{0]}^{Q^{\varphi}}\left\{\frac{\varphi(w(0))}{\varphi(w(t))} \cdot \exp \int_{0}^{t} \frac{\Delta \varphi}{2 \varphi}\left(w_{s}\right) d s+t F_{\delta}(t)\right\}
$$

$\geq$ (by Jensen's inequality)

$\geq \liminf \frac{1}{t}\left\{E_{0]}^{Q^{\varphi}}\left(\log \varphi\left(w_{0}\right)-E_{0]}^{Q^{\varphi}}\left(\log \varphi\left(w_{t}\right)\right)+E_{0]}^{Q^{\varphi}}\left(\int_{0}^{t} \frac{\Delta \varphi}{2 \varphi}\left(w_{s}\right) d s+F_{\delta}(t)\right)\right\}\right.$

where we have used the notation

$$
F_{\delta}(t)=2 \alpha P_{t} T_{[0 t]}\left(\int_{0}^{\infty} \frac{e^{-\sigma} d \sigma}{\left|w_{\sigma}-w_{0}\right|+\delta}\right)
$$

Now by definition (cf. (3.3)) $\varphi$ belongs to the Sobolev space $W^{1,2}\left(\mathbb{R}^{3}\right)$ hence by Sobolev inequality (cf. [ ], Theorem (IX.9)) $\varphi \in L^{6}\left(\mathbb{R}^{3}, d x\right)$. Therefore, since by definition $\log \varphi \in L^{2}\left(\mathbb{R}^{3}, d x\right)$, a simple argument allows to conclude that $\varphi \in L^{2} \log L\left(\mathbb{R}^{3}, d x\right)$, hence

$$
E_{0]}^{Q^{\varphi}}\left(\log \varphi\left(w_{t}\right)\right)<+\infty ; \quad \varphi^{2}(x) d x-\text { a.e. } ; \forall t \geq 0
$$

Then

$$
\begin{gathered}
E_{x}^{w}\left(\exp t F_{\delta}\left(P_{t} T_{[0, t]}\right)\right)=E_{x}^{Q_{\phi}}\left(\frac{\phi(x)}{\phi(w(t))} \exp \left(\int_{0}^{t} \frac{\Delta \phi}{2 \phi}(w(s)) d s+t F_{\delta}\right)\right. \\
\geq \frac{\phi(x)}{\sup \phi} \exp \left[E_{x}^{P_{\phi}}\left(\int_{0}^{t} \frac{\Delta \phi}{2 \phi}(w(s)) d s\right)+t E_{x}^{Q_{\phi}}\left(F_{\delta}\left(P_{t} T_{[0, t]}\right)\right)\right] \\
\liminf _{t \rightarrow \infty} \frac{1}{t} \log E_{x}^{w}\left(\exp t F_{\delta}\left(P_{t} T_{[0, t]}\right)\right) \geq \\
\geq \liminf _{t \rightarrow \infty} E_{x}^{Q_{\phi}}\left(\frac{1}{t} \int_{0}^{t} \frac{\Delta \phi}{2 \phi}(w(s)) d s\right)+\liminf _{t \rightarrow \infty} E_{x}^{Q_{\phi}}\left(F_{\delta}\left(P_{t} T_{[0, t]}\right)\right)
\end{gathered}
$$


But one has, $\omega-Q^{\phi}-$ a.e.

$$
\begin{gathered}
\lim _{t \rightarrow \infty} \frac{1}{t} \int_{0}^{t} \frac{\Delta \phi}{2 \phi}(\omega(s)) d s=\int_{\mathbb{R}^{3}} \frac{\Delta \phi}{2 \phi} \phi^{2} d x= \\
=\frac{1}{2} \int_{\mathbb{R}^{3}} \phi \Delta \phi d x=-\frac{1}{2} \int_{\mathbb{R}^{3}}|\nabla \phi|^{2} d x
\end{gathered}
$$

Moreover by the ergodic theorem, as $t \rightarrow \infty$

$$
\begin{gathered}
F_{\delta}\left(P_{t} T_{[0, t]}\right)(\omega)=2 \alpha \int_{0}^{t} d s \int_{0}^{\infty} \frac{e^{-\sigma} d \sigma}{\delta+\left|\omega_{t}(\sigma)-\omega_{t}(0)\right|} \rightarrow \\
Q^{\phi}\left(2 \alpha \int_{0}^{\infty} \frac{e^{-\sigma} d \sigma}{\delta+|\omega(\sigma)-\omega(0)|}\right) \omega-Q-\text { a.e. }
\end{gathered}
$$

Then

$\liminf \frac{1}{t} \log E^{w}\left(\exp t F_{j}\left(P_{t} T_{[0, t]}\right)\right) \geq Q^{\phi}\left(2 \alpha \int_{0}^{\infty} \frac{e^{-\sigma} d \sigma}{\delta+|\omega(\sigma)-\omega(0)|}\right)-\int_{\mathbb{R}^{3}}|\nabla \phi|^{2} d x$

$$
\Rightarrow \forall \delta>0, \quad \forall \phi \in H_{c}, \quad \forall x \in \operatorname{supp} \phi
$$

$$
\begin{aligned}
& \liminf _{t \rightarrow \infty} \frac{1}{t} \log E_{x}^{w}\left(\exp \alpha \int_{0}^{t} \int_{0}^{t} \frac{e^{-|\sigma-\tau|} d \tau d \tau}{|\omega(\sigma)-\omega(\tau)|}\right) \geq \\
& \geq Q^{\phi}\left(2 \alpha \int_{0}^{\infty} \frac{e^{-\sigma} d \sigma}{|\omega(\sigma)-\omega(0)|+\delta}\right)-\int_{\mathbb{R}^{3}}|\nabla \phi|^{2} d y
\end{aligned}
$$

Since

$$
\frac{1}{|\omega(\sigma)-\omega(0)|+\delta} \leq \frac{1}{|\omega(\sigma)-\omega(0)|} \in L^{1}\left(Q^{\phi}\right) \quad \forall \delta>0
$$

by dominated convergence one has:

$$
\begin{gathered}
\Rightarrow \lim _{\delta \rightarrow 0} Q^{\phi}\left(\frac{1}{\mid \omega(\sigma)-\omega(0) 1+\delta}\right)=Q^{\phi}\left(\frac{1}{\mid \omega(\sigma)-\omega(0)}\right) \\
\Rightarrow \underline{\lim } \frac{1}{t} \log E_{x}^{w}\left(\exp \alpha \iint \frac{e^{-|\sigma-\tau|} d \tau d \iota}{|\omega(\sigma)-\omega(\tau)|}\right) \geq \\
\geq Q^{\phi}\left(2 \alpha \int_{0}^{\infty} \frac{e^{-\sigma} d \sigma}{|\omega(\sigma)-\omega(0)|}\right)-\int_{\mathbb{R}^{3}}|\nabla \phi|^{2} d y
\end{gathered}
$$


Let $\tau_{h} \phi(y)=\phi(y+h)$ then

$$
\int_{\mathbb{R}^{3}}\left|\nabla\left(\tau_{h} \phi\right)\right|^{2} d y=\int_{\mathbb{R}^{3}}|\nabla \phi|^{2} d y \quad \forall h \in \mathbb{R}^{3}
$$

If it is possible to show that

$$
Q^{\phi}\left(2 \alpha \int_{0}^{\infty} \frac{e^{-\sigma} d \sigma}{|\omega(\sigma)-\omega(0)|}\right)=Q^{\tau_{h} \phi}\left(2 \alpha \int_{0}^{\infty} \frac{e^{-\sigma} d \sigma}{|\omega(\sigma)-\omega(0)|}\right)
$$

Then the expression on the right is translational invariant, then $\forall \phi \in H_{c}$

$\underline{\lim } \frac{1}{t} \log E^{w}\left(\exp \alpha \int_{0}^{t} \int_{0}^{t} \frac{e^{-\mid \sigma-\tau}}{|\omega(\sigma)-\omega(\tau)|}\right) \geq Q^{\phi}\left(2 \alpha \int_{0}^{\infty} \frac{e^{-\sigma} d \tau}{|\omega(\sigma)-\omega(0)|}\right)-\int_{\mathbb{R}^{3}}|\nabla \phi|^{2} d m$ and the thesis follows as in the proof of LB1.

$$
\begin{gathered}
F_{\delta, \lambda}(\omega)=2 \alpha \int_{0}^{\infty} \frac{\lambda e^{-\lambda \sigma}}{Q \in M_{s}(\Omega) \mapsto Q\left(F_{\delta, \lambda}\right) \text { bounded and continuous }} \\
\frac{1}{t} \int_{0}^{t} F_{\delta}\left(\theta_{s} \omega_{t}\right) d s=\frac{2 \alpha}{t} \int_{0}^{t} d s \int_{0}^{\infty} \frac{\lambda e^{-\lambda t} d \sigma}{\left.\left(\delta^{2}+\mid \omega_{t}(\sigma+s)-\omega_{t}(s)\right)\right)^{1 / 2}}= \\
=\frac{2 \alpha}{t} \int_{0}^{t} d s\left[\int_{0}^{t-s} \frac{\lambda e^{-\lambda \sigma}}{\left(\delta^{2}+\left|\omega_{t}(\sigma+s)-\omega_{t}(s)\right|\right.} d \sigma+\right. \\
+\int_{t-s}^{\infty} \frac{\lambda e^{-\lambda \sigma} d \sigma}{\left.\left(\delta^{2}+\left|\omega_{t}(\sigma+s)-\omega_{t}(s)\right|^{2}\right)^{1 / 2}\right]} \sigma^{\prime}=\sigma+s \\
\int_{0}^{t} F_{\delta}\left(\theta_{s} \omega_{t}\right) d s-2 \alpha \int_{0}^{t} d s\left[\int_{0}^{t} \frac{\lambda e^{-\lambda(\sigma-s)} d \sigma}{\left(\left|\omega_{t}(\sigma)-\omega_{t}(s)\right|^{2}+\delta^{2}\right)^{1 / 2}} \mid=\right. \\
=\left|\int_{0}^{t} F_{\delta}\left(\theta_{s} \omega_{t}\right) d s-\alpha \int_{0}^{t} \int_{0}^{t} \frac{\lambda e^{-\lambda|\sigma-s|} d \sigma d s}{\left(\delta^{2}+|\omega(\sigma)-\omega(s)|^{2}\right)^{1 / 2}}\right| \leq \\
\leq \frac{2 \alpha}{\delta} \int_{0}^{t} d s \int_{t-s}^{\infty} \lambda e^{-\lambda \sigma} d \sigma=\frac{2 \alpha}{\delta} \int_{0}^{t} d s \int_{t-s}^{\infty} e^{-\lambda \sigma} d \sigma= \\
=\frac{2 \alpha}{\delta} \int_{0}^{t} d s\left[e^{-\lambda(t-s)}\right]=\left.\frac{2 \alpha}{\delta} e^{-\lambda t} \frac{1}{\lambda} e^{\lambda s}\right|_{0} ^{t}=\frac{2 \alpha}{\delta \lambda}\left(1-e^{\lambda t}\right) \leq \frac{2 \alpha}{\delta \lambda} \\
\left.-\left.\frac{e^{-\lambda \sigma}}{\lambda}\right|_{t-s} ^{\infty}\right]=
\end{gathered}
$$

